\title{
¿Jugar o aprender? El aprendizaje lúdico en la formación musical del maestro
}

\author{
Miquel Alsina Tarrés ${ }^{1}$; Ivet Farrés Cullell ${ }^{2}$
}

Recibido: 17 de marzo de 2020 / Aceptado: 1 de febrero de 2021

Resumen. Los diversos modos y estrategias del aprendizaje lúdico (juego, gamificación, simulación, etc.) se han convertido en elementos de innovación metodológica de uso creciente en la docencia universitaria. En este artículo se presenta el estudio sobre una práctica de educación musical ludificada, llevada a cabo en una materia troncal de educación artística del Grado de Maestro en Educación Primaria, con un grupo de alumnos de segundo curso $(n=90)$ de una universidad española. La práctica se realiza en grupos de cinco alumnos, a lo largo de seis semanas, y la aplicación final del proyecto se realiza con escolares de Primaria de Ciclo Medio y Superior. Siguiendo una metodología de estudio de caso, con análisis de datos cualitativos y cuantitativos, se codifican y evalúan diferentes aspectos de las actividades diseñadas, con el fin de analizar: a) las interacciones entre participantes, b) los contenidos musicales curriculares, y c) las estrategias de ludificación empleadas. Los resultados revelan un alto grado de implicación y colaboración, adecuación a los contenidos musicales curriculares, y predilección por estrategias de juego ligadas a las clasificaciones, la obtención de feedback y los desafíos.

Palabras clave: aprendizaje lúdico; didáctica de la música; maestro de Primaria; formación docente.

\section{[en] Playing or learning? Playful learning in teacher's musical training}

Abstract. The various methods and strategies of playful learning (game, gamification, simulation, etc.) have become elements of methodological innovation increasingly used in university teaching. This article presents the study on a gamified musical education practice, carried out in a core subject of artistic education of the Degree in Primary Education, with a group of second-year students $(n=90)$ from a Spanish university. The application is carried out in groups of five students, over six weeks, and the final implementation of the project is carried out with primary school students from the Middle and the Upper Cycle (8 to 11). Following a mixed, qualitative and quantitative, case study methodology, different aspects of the designed activities are coded and evaluated in order to be analyzed: a) the interactions among participants, b) the curricular musical contents, and c) the gamification strategies used. The results reveal a high degree of involvement, collaboration and adaptation to the curricular musical content, moreover, they underline game strategies linked to classification, obtaining feedback and challenges.

Keywords: playful learning; music pedagogy; primary education; teacher training.

Sumario. 1. Introducción. 2. Fundamentación teórica. 3. Metodología y objetivos de la investigación. 4. Exposición de resultados. 5. Discusión y conclusiones. 6. Aplicación y limitaciones del estudio. 7. Referencias bibliográficas.

Cómo citar: Alsina, M.; Farrés, I. (2021). ¿Jugar o aprender? El aprendizaje lúdico en la formación musical del maestro. Revista Electrónica Complutense de Investigación en Educación Musical, 18, 83-96. http://doi.org/10.5209/reciem.67853

\section{Introducción}

A priori, jugar y aprender no tienen por qué ser ni opuestos ni excluyentes. Aun así, a medida que avanzamos en las edades y etapas escolares, lo uno y lo otro parece distanciarse en el imaginario colectivo, de manera que el acto del juego y del estudio ocupan momentos y funciones diferenciados. Son numerosos los autores y corrientes pedagógicas

\footnotetext{
Universitat de Girona (España). Departamento de Didácticas Específicas

E-mail: miquel.alsina@udg.edu

https://orcid.org/0000-0001-6625-1097

2 Universitat de Girona (España). Departamento de Didácticas Específicas

E-mail: ivet.farres@udg.edu

https://orcid.org/0000-0003-2179-3022
}

Rev. electrón. complut. inves. educ. music. 18, 2021: 83-96 
que han vinculado el juego con el aprendizaje, muy especialmente en el contexto de la educación infantil (Hassiger-Das et al., 2017; Zich, Ortega-Ruiz \& Sibaja, 2016), también en las demás etapas educativas, bajo el concepto genérico y modelizado de aprendizaje lúdico o gamificado (Garris, Ahlers \& Driskell, 2002). Así se recogía en el informe Horizon Report: 2014 K-12 (Johnson, Adams Becker, Estrada \& Freeman, 2014), que planteaba la incorporación progresiva de aspectos de la dinámica del juego en contextos no lúdicos para ayudar a potenciar la implicación de los estudiantes. Revisiones sistemáticas sobre el concepto, como el estudio genérico de Ortiz-Colón, Jordán y Agredal (2018), o los referidos explícitamente a las prácticas de gamificación o uso del juego en la formación universitaria, en Lozada y Betancur (2017) y Prieto (2020), indican un interés continuo y creciente sobre la cuestión.

También la era de la tecnología y la sociedad multimedial del siglo XXI han perfilado una nueva dimensión del juego (videojuegos, juegos en línea, etc.) en la que el niño ha perdido libertad y espacio para la propia iniciativa (Elkind, 2008). Cambios que, en una sociedad tendente a la uniformidad y a la globalización, constituyen elementos suficientes, desde una perspectiva crítica con estos "avances", para hablar de una redefinición de la infancia y de la niñez (Bautista \& Guzmán, 2003; Tulloch \& Randell-Moon, 2018).

Un mundo global e interconectado conlleva para sus habitantes la compartición de modas, gustos, conductas y valores. Disfrutar de los mismos logros y padecer las mismas carencias o enfermedades. Algunos autores han llegado a la constatación de que vivimos en un mundo esencialmente ludificado (gamefull world), en el que, no solo jugamos los mismos juegos, sino que, a demás, consumimos y participamos en una inducida e interesada confusión entre realidad y ficción (Deterding, 2014). En el ámbito de la ludificación, el mundo educativo ha desarrollado técnicas y tendencias igualmente diversas y, en cierto modo, híbridas y ambiguas.

La vinculación entre la educación musical y el juego tiene ya un largo recorrido. Entre otros muchos campos de indagación, interesa a la didáctica de la música analizar datos y resultados sobre los beneficios de incorporar dinámicas colaborativas como, por ejemplo, las afines al juego grupal. Elementos, colaboración y juego, vinculados frecuentemente al despliegue de la creatividad, y a prácticas de co-creación que han probado su incidencia en el desarrollo de competencias para el trabajo en equipo, tales como la identificación de objetivos comunes, el compromiso, la comunicación, el liderazgo, la socialización, y la identidad colectiva (Carrión, 2019; Criss, 2010).

En este artículo se plantea como objetivo principal valorar, desde el contexto universitario de la instrucción musical y didáctica del maestro de Educación Primaria, en qué medida las estrategias de ludificación o simulación del juego resultan efectivas en el desarrollo de competencias docentes de la materia. Para ello se investiga, a través de un estudio de caso, cuales son las aportaciones formativas y características metodológicas predominantes en una práctica académica determinada.

\section{Fundamentación teórica}

\subsection{Aprendizaje lúdico: juego y gamificación}

En el aprendizaje lúdico, el aprendizaje se da principalmente porque los niños (o adultos) están activos, involucrados, e interactuando socialmente. La actividad en si misma les atrae y el tema les seduce y capta su atención (Hirsh-Pasek et al., 2015). Por esa misma razón, en algunos autores se afirma que, más que contrarios, conceptualmente hablando, juego y aprendizaje son términos complementarios o equivalentes (Hirsh-Pasek \& Golinkoff, 2008). De hecho, desde ámbitos como la pedagogía y la psicología, se reivindica el juego no solo como fuente de motivación y predisposición hacia el aprendizaje, sino, además, como potente herramienta de desarrollo intelectual, emocional y social, con efectos positivos a largo plazo sobre las capacidades de aprendizaje y desarrollo integral del individuo (Perdomo \& Rojas, 2019; Singer, Golinkoff \& Hirsh-Pasek, 2006).

En los contextos de aprendizaje lúdico cabe distinguir, de entrada, entre los procesos de gamificación y el uso del juego como tal, en sus modalidades de juego libre, juego guiado (o semiguiado) por el docente, y juego reglado. En las dos primeras modalidades de juego, resulta conveniente que sea el alumno el que autogestione las fases y grados de implicación en la actividad e imponga sus propias normas, de modo que el profesor intervenga únicamente de forma puntual, evitando asumir el control absoluto de la dinámica (Weisberg, Hirsh-Pasek, Golinkoff, Kittredge \& Klahr, 2016).

El juego reglado, en cambio, dispone de unas reglas claras. Durante su desarrollo el docente acostumbra a controlar explícitamente la actividad, gestionando las normas del propio juego, los participantes comparten el control cuando llega su turno, junto a fases o momentos sujetos a la improvisación y al azar. En cualquiera de sus modalidades, el juego ha sido utilizado para la integración de contenidos curriculares en diferentes momentos y contextos educativos muy variados, fomentando los aprendizajes en un entorno participativo, interesante, significativo y socialmente interactivo (Hassinger-Das et al., 2017).

El término "gamificación" fue acuñado por primera vez en el año 2002 por el programador informático británico Nick Pelling, creador de videojuegos como Arcadians (1982) o Frank! (1984), y propietario de una importante consultoría (Conundra), especializada en la conjugación de la electrónica, la informática y el juego. El término es también equivalente al de "ludificación", entendido genéricamente como el uso consciente de elementos propios del juego en contextos diferentes a este (Robson, Plangger, Kietzmann, McCarthy \& Pitt, 2015). 
Desde que fue concebida como tal, la gamificación se ha aplicado conscientemente en ámbitos tan diversos como el marketing, la política, las ciencias médicas, los negocios online y, por supuesto, en la educación y la formación profesional. La estrategia subyacente en la gamificación es la de rentabilizar los elementos positivos, tales como la motivación, la competencia, la socialización, etc., que en general afloran en el momento en el que enmarcamos una situación determinada bajo los parámetros del juego (Lieberoth, 2015). Para ello se han catalogado una serie de estrategias y mecanismos propios del juego que a priori funcionan como motores de esas conductas o respuestas asociadas. Entre los recursos más habituales, destacan aspectos como los niveles de logro, retos o pruebas, sistema de recompensas, uso de avatares, relaciones de competición, cooperación y colaboración entre usuarios, y uso de la narrativa (Borrás, 2015; Teixes, 2014).

Algunas de las técnicas principales para los procesos de ludificación se reconocen también en inglés tras las siglas PBL: Points, Badges and Leaderboards (Puntos, Medallas o Insignias, y Clasificaciones), a las que puede añadirse elementos de índole motivacional o competitivo, como niveles, regalos, desafíos y retos. Finalmente, otra categoría de elementos también presentes en las estrategias de gamificación son las recompensas ligadas al placer o la estética, de tipo hedonista y vinculadas a la experiencia psicosensorial: sensaciones, fantasías, complicidades, y conocimiento del otro y de uno mismo, entre otras (Werbach \& Hunter, 2014).

A diferencia del juego como tal, la experiencia de gamificar en contextos educativos supone trasladar o adaptar mecánicas del juego (especialmente del videojuego) a secuencias didácticas, con el fin de obtener una mejora en el proceso y en los resultados del aprendizaje, con o sin participación de elementos tecnológicos (Deterding, 2013; Deterding, Dixon, Khaled, \& Nacke, 2011). Algunos de los elementos más utilizados del (video)juego en su adaptación al aula son los estadios y progresiones visuales, la competición y cooperación con la idea de recurrir a un compromiso social, la libertad de elección, la libertad de fracasar y la retroalimentación o feedback en un tiempo breve (véase, como ejemplo, la adaptación del concepto de Escape Room en García-Lázaro, 2019). Aspectos a los que se les añade, frecuentemente, el desarrollo de la narrativa, entendida como dimensión que articula y cataliza la acción (Contreras, 2017). La finalidad es, en resumen, acercar los contenidos al alumnado de una forma divertida y motivadora, en un espacio donde ellos son los principales agentes activos: están involucrados y se divierten mientras cumplen objetivos educativos. Se podría concluir que, en la gamificación, el docente se convierte en un diseñador de actividades lúdicas promotoras del aprendizaje, mientras que el estudiante asume el rol de usuario-jugador (Ardila-Muñoz, 2019).

\subsection{El juego en la educación musical}

La orientación lúdica es una de las características distintivas de buena parte de las metodologías o propuestas metodológicas (activas, instrumentales, creativas...) surgidas en educación musical a lo largo del siglo XX (Hemsy de Gainza, 2004). El juego, por ejemplo, es un importante componente en las propuestas del pedagogo y compositor húngaro Zoltán Kodály (1882-1967), cuyo desarrollo supone la sustitución del solfeo académico por una nueva dimensión lúdico-social del aprendizaje musical, sin renunciar a la precisión ni fluidez de la práctica vocal (Gault, 2016). Método, el de Kodály, de amplio uso y aceptación en contextos de formación del profesorado (Lucato, 2001). Junto a este referente particular, son el conjunto de pedagogos, aun hoy de referencia (Orff, Willems, Dalcroze, etc.), los que se alinean históricamente con los primeros postulados del constructivismo y su consiguiente evolución (Navarro, 2017).

Hein (2014) resume los juegos musicales más habituales en tres categorías: los vinculados a ejercicios de memoria (drill and skill), los juegos rítmicos, y las propuestas lúdico-educativas en torno a juguetes musicales. En sintonía con el constructivismo, la gamificación en la didáctica musical supone una herramienta más para la implementación del aprendizaje de la música de manera activa, centrada en el alumno, atendiendo al punto de partida o estado inicial de cada individuo, y haciendo hincapié en sus centros de interés (Clapper, 2014). Así mismo, como sucede en las otras materias del currículo, un enfoque de la educación musical arraigada en el constructivismo ejerce, a priori, mayor incidencia sobre el desarrollo emocional, intelectual, social y físico del sujeto, y, por tanto, en su desarrollo integral (Scott, 2009). Disponemos de evidencias en el campo de la educación musical que reseñan los beneficios de enfoques metodológicos afines al aprendizaje lúdico (Ray, 2008; Scott, 2009, 2011; Shively, 2015). Desde la perspectiva de la formación inicial, Cleaver y Ballantyne (2013) ponen de relieve cómo, en el ámbito de la educación musical, la perspectiva constructivista del docente viene moldeada por elementos subjetivos (tales como su experiencia y tendencias musicales, su ideología y sus creencias, su autobiografía formativa, etc.), con igual o mayor peso que el de la formación académica recibida como futuro maestro. En cualquier caso, diremos con Restrepo (2006), que la visión favorable del docente en pos de un enfoque constructivista es un elemento imprescindible dentro del propio sistema educativo para que este experimente un verdadero desarrollo.

Como se ha afirmado ya, las prácticas de gamificación se originan, y mantienen una estrecha relación, con el mundo del videojuego y otras ofertas lúdicas de las industrias digitales. En un estimulante ejercicio, Politis, Margounakis, Aleksii, \& Karanikas (2017) han analizado bajo la mirada de la sinestesia la relación entre la música, o conjunto de estímulos sonoros, y el desarrollo visual-narrativo del videojuego, incluyendo la perspectiva del aprendizaje gamificado. También irrumpe, en este entorno, el concepto de serious game, es decir, videojuego creado con finalidades formativas específicas (véase un ejemplo centrado en la formación del canto infantil en García-Hernández et al., 2014). La mayor parte de experiencias documentadas sobre gamificación en educación musical, mantienen esta filiación 
con las tecnologías. Por ejemplo, en contextos de educación general, para el abordaje de partes del currículo con un enfoque innovador, con resultados que subrayan la aportación de la gamificación al factor motivacional e incidencia en aprendizajes competenciales transversales (Gomes, Figueiredo \& Amante, 2014; Gomes, Figueiredo, y Bidarra, 2014). Otros estudios se centran específicamente en prácticas de gamificación aplicadas al aprendizaje instrumental, por ejemplo, en estudiantes de violín (Margoudi, Oliveira \& Waddell, 2016; Margoudi, Waddell \& Oliveira, 2017). $\mathrm{O}$, finalmente, se reportan experiencias y resultados del uso de herramientas tecnológicas creadas para el trabajo de las capacidades musicales de los usuarios, por ejemplo, en el caso de la celebrada plataforma en línea Troubadour para la formación del oído musical a partir de actividades gamificadas de autoformación (Pesek, Vucko, Savli, Kavcic \& Marolt, 2020).

Finalizamos el apartado con la estimulante referencia al concepto del flow, acuñado y desarrollado por Csikszentmihalyi (1990, 2000), como estado resultante en el individuo de la dedicación plena y disfrute de la actividad que realiza, también, especialmente, en contextos lúdicos. Así, el flow ha sido identificado como uno de los ingredientes placenteros y facilitadores del aprendizaje en entornos gamificados (Hamari \& Koivisto, 2014). Ya en la arena musical, el concepto se vincula o identifica con aspectos ligados a la fluidez, el ritmo, la creatividad y la inspiración en el músico, creador o intérprete (Burnard, 2012). Carolyn Wagner, por ejemplo, ha desarrollado reflexiones y propuestas de transferibilidad a la pedagogía del piano de algunos de los elementos desencadenantes del flow que experimenta el participante en un videojuego (Wagner, 2017).

\section{Metodología y objetivos de la investigación}

La metodología de esta investigación es de tipo descriptiva-interpretativa, siguiendo un paradigma mixto, cualitativo-cuantitativo, a partir de un estudio de campo en el que se indaga sobre una práctica formativa determinada (Rio Sadornil, 2003). Los datos se recogen y analizan desde la posición de observador participante, como docentes de una asignatura troncal de educación musical del Grado de Educación Primaria. Los individuos de la muestra se componen de una agrupación regular de alumnos de segundo curso $(\mathrm{N}=90)$, divididos en tres subgrupos de tamaño similar (A1, A2 y A3), que cursan dicho grado en una universidad española. En cada uno de los tres subgrupos se forman aleatoriamente agrupaciones de trabajo de cinco componentes. Este paradigma ofrece la posibilidad de mantener la práctica regular en el aula y actuar, a su vez, como docente-investigador, sin desnaturalizar la dinámica habitual del curso ni la configuración de alumnos de los grupos clase preestablecidos, presentando finalmente un informe del proceso que responda a las cuestiones planteadas (Creswell \& Creswell, 2018). En este sentido, el papel de los docentes como investigadores participantes se aproxima a los esquemas de la investigación acción, sin llegar a serlo por falta de ciclos reflexivos recurrentes. Aún así, el proceso seguido comparte con esta metodología una fuerte incidencia del componente colaborativo (equipo docente, grupos de alumnos, facultad-escuela) y la voluntad de examinar el propio trabajo para mejorarlo (Cain, 2013).

La investigación se organiza en torno a estas tres preguntas indagatorias:

- ¿Qué dinámicas de interacción se producen entre los participantes (futuros maestros y escolares) en las actividades musicales ludificadas?

- ¿Qué tipo de contenidos musicales se trabajan en las actividades diseñadas?

- ¿Cuáles son las estrategias de ludificación y los grados de preferencia en las prácticas diseñadas por los maestros en formación?

Se investiga pues sobre los resultados de una práctica en la que los alumnos del grado reciben una formación inicial y, a continuación, siguen las directrices para la co-creación de una actividad de simulación, con elementos del juego, adecuada al currículo vigente de educación musical en Primaria (Catalunya, 2015) y los diferentes elementos concretados en el desarrollo competencial del Área de Educación Artística (Generalitat de Catalunya, 2016). Se trata de una práctica instaurada en la asignatura durante los últimos cuatro cursos académicos, que concluye, en la última sesión de trabajo, con la realización de las actividades en una de las escuelas de Educación Primaria colaboradoras en el prácticum del Grado a la que se traslada la actividad. Para el proyecto descrito se limitan los recursos tecnológicos al uso de dispositivos para la reproducción de sonido o visionado de imágenes complementarias a la actividad. La justificación de esta limitación es la voluntad de los docentes de que los alumnos utilicen prioritariamente sus recursos corporales e instrumentales (voz, percusión, movimiento, etc.), así como otros materiales de creación propia, apartándose así del concepto de gamificación en sentido estricto, ligado y restringido al videojuego y al uso de las tecnologías, adoptando como alternativa el constructo de "actividad lúdica de aprendizaje".

Conceptualmente, la práctica académica desarrolla también la idea de "evento de interacción formativa" o Interaction Learning Event (ILE), formulado por Karl M. Kapp como paradigma propio de los procesos o propuestas educativas ligados al juego, la gamificación, y la simulación (Kapp, 2012). Según el mismo autor, las estrategias didácticas en torno a prácticas docentes de esta tipología se justifican cuando se atienda a alguno de los objetivos relacionados en la Tabla 1, ligados a aspectos de motivación, práctica reflexiva, intervención conductual, y ganancia 
experiencial. En la tabla también se asigna a cada objetivo necesidades formativas en relación con las competencias profesionales a desarrollar por los maestros en formación a lo largo de la práctica.

Tabla 1. Objetivos genéricos de los ILE (Kapp, 2012; Kapp, Blair \& Mesch, 2014)

y su conexión con los objetivos docentes de la investigación

\begin{tabular}{|l|l|}
\hline Objetivos del formato ILE & Necesidades formativas del Grado \\
\hline Superar la desmotivación & $\begin{array}{l}\text { Ganar implicación y participación de los maestros en } \\
\text { formación diseñando y creando la actividad musical }\end{array}$ \\
\hline $\begin{array}{l}\text { Facilitar oportunidades para el pensamiento profundo } \\
\text { y la reflexión }\end{array}$ & $\begin{array}{l}\text { Generar discusión a lo largo de un proceso de elabora- } \\
\text { ción didáctica conjunta }\end{array}$ \\
\hline Cambiar conductas en positivo & $\begin{array}{l}\text { Superar prejuicios y empoderar al alumnado en rela- } \\
\text { ción con el uso del lenguaje musical }\end{array}$ \\
\hline Promover prácticas auténticas & $\begin{array}{l}\text { Ejercitar el rol docente en situaciones próximas al con- } \\
\text { texto profesional real }\end{array}$ \\
\hline
\end{tabular}

El proceso se desarrolla a lo largo de cuatro fases, durante seis semanas y doce sesiones de noventa minutos. En la primera fase, se ofrece una formación de dos sesiones sobre los orígenes, características y ejemplos de propuestas ludificadas en educación musical (con y sin tecnología) que recibe todo el grupo clase (N=90). En la segunda fase, creados los grupos de forma aleatoria, se encarga el diseño y elaboración de una actividad de aprendizaje musical que debe responder a alguno de los parámetros del aprendizaje basados en el juego. Para asegurar la diversidad de propuestas, se asigna ámbitos disciplinares distintos (melodía, ritmo, timbre, y cuerpo-danza). En la tercera fase, se aplica la propuesta entre los mismos alumnos del grado, lo que sirve para introducir mejoras significativas, principalmente referidas a las reglas y desarrollo de la actividad. La cuarta y última fase consiste en la aplicación de todas las actividades a los alumnos de ciclo medio y superior de una escuela de Educación Primaria $(\mathrm{N}=106)$. Este enfoque añade una segunda dimensión a la simulación, en la que los alumnos universitarios asumen el rol de maestros y actúan en consecuencia. Para la evaluación de los estudiantes universitarios se elabora una tabla de evaluación competencial consensuada con el equipo docente de la asignatura (véase Anexo 1).

Los datos referidos al objetivo del estudio y a las preguntas a investigar se obtienen del visionado de las grabaciones realizadas durante la puesta en práctica de las actividades en la escuela, y del trabajo (descriptivo, reflexivo y autoevaluativo) presentado por escrito por cada grupo de alumnos. Las grabaciones se analizan con la ayuda del software ATLAS.ti 8, para el análisis cualitativo de los datos. Para este proceso se adoptan seis categorías de observación de una taxonomía propuesta por Kapp, Blair y Mesch (2014: 57-58) [(véase Figura 1)]. A partir de estas categorías matrices se procede a la codificación del material grabado, lo que supone el análisis de un total de quince actividades diseñadas y puestas en práctica por los grupos de estudiantes de magisterio. En la tabla siguiente [2] se ofrecen, a modo de ejemplo, las características principales de las actividades diseñadas por los cinco grupos de trabajo pertenecientes al grupo-clase A1.

Tabla 2. Información resumida de las cinco actividades elaboradas por el grupo A1 de la muestra

\begin{tabular}{|l|l|l|l|}
\hline $\begin{array}{l}\text { Título de la activi- } \\
\text { dad/juego musical }\end{array}$ & $\begin{array}{l}\text { Dimensiones curri- } \\
\text { culares (*) }\end{array}$ & $\begin{array}{l}\text { Contenidos musi- } \\
\text { cales }\end{array}$ & Sinopsis de la narrativa del juego o simulación \\
\hline La Isla del Silencio & $\begin{array}{l}\text { Percepción y escu- } \\
\text { cha. } \\
\text { Expresión, interpre- } \\
\text { tación y creación. }\end{array}$ & $\begin{array}{l}\text { Altura del sonido y } \\
\text { afinación. }\end{array}$ & $\begin{array}{l}\text { Los participantes se encuentran en la Isla del Si- } \\
\text { lencio, donde yace dormida y captiva de un pirata, } \\
\text { la Reina del Silencio. A partir de una serie de } \\
\text { pistas, los participantes deben transcribir las notas } \\
\text { musicales de una canción con la que despertar y } \\
\text { salvar la reina. }\end{array}$ \\
\hline $\begin{array}{l}\text { El misterio de las } \\
\text { notas perdidas }\end{array}$ & $\begin{array}{l}\text { Interpretación y } \\
\text { producción. }\end{array}$ & $\begin{array}{l}\text { Figuras musicales y } \\
\text { ritmo. }\end{array}$ & $\begin{array}{l}\text { Un director de orquestra sufre el robo de sus parti- } \\
\text { turas la noche antes de un concierto que debe diri- } \\
\text { gir. Los participantes han de reconstruir los ritmos } \\
\text { de la música para ayudarle a recordar la obra del } \\
\text { concierto. }\end{array}$ \\
\hline El reto de Li Blu & $\begin{array}{l}\text { Percepción, com- } \\
\text { prensión y valora- } \\
\text { ción. } \\
\text { Interpretación y } \\
\text { producción. }\end{array}$ & $\begin{array}{l}\text { El timbre y los } \\
\text { instrumentos musi- } \\
\text { cales. }\end{array}$ & $\begin{array}{l}\text { Los participantes leen una carta del personaje } \\
\text { mágico del juego, Li Blu, en la que invita a los } \\
\text { participantes a concursar para superar cuatro retos, } \\
\text { de dificultad creciente, en torno a la identificación } \\
\text { auditiva de los instrumentos musicales. }\end{array}$ \\
\hline
\end{tabular}




\begin{tabular}{|l|l|l|l|}
\hline Trivial musical & $\begin{array}{l}\text { Percepción, com- } \\
\text { prensión y valora- } \\
\text { ción. } \\
\text { Interpretación y } \\
\text { producción. }\end{array}$ & $\begin{array}{l}\text { Los participantes se dividen en dos equipos que } \\
\text { interpretación meló- } \\
\text { dica de una canción. }\end{array}$ & $\begin{array}{l}\text { leben competir para sumar puntos respondiendo a } \\
\text { preguntas, organizadas en tres niveles de dificul- } \\
\text { tad. El equipo ganador puede elegir qué canción } \\
\text { escuchar y cantar con soporte de una versión en } \\
\text { karaoke. }\end{array}$ \\
\hline Perdidos en la selva & $\begin{array}{l}\text { Interpretación y } \\
\text { producción. }\end{array}$ & $\begin{array}{l}\text { Altura y timbre del } \\
\text { sonido. Identifica- } \\
\text { ción auditiva de los } \\
\text { instrumentos musi- } \\
\text { cales. }\end{array}$ & $\begin{array}{l}\text { Se traslada un grupo de exploradores a la selva. } \\
\text { Antes de acampar, deben descartar que haya algún } \\
\text { animal peligroso. Divididos en dos grupos que } \\
\text { compiten entre ellos, deben identificar los soni- } \\
\text { dos de algunos animales y representarlos con el } \\
\text { cuerpo, asociándolos posteriormente al timbre de } \\
\text { instrumentos musicales. }\end{array}$ \\
\hline
\end{tabular}

(*) A partir del documento curricular vigente (Generalitat de Catalunya, 2016)

En el proceso de codificación se ha practicado el método descriptivo (descriptive coding o topic coding), identificando y agrupando en el proceso qué (inter)acciones protagonizan las diversas fases de la puesta en práctica de cada actividad (Saldana, 2016: 102).

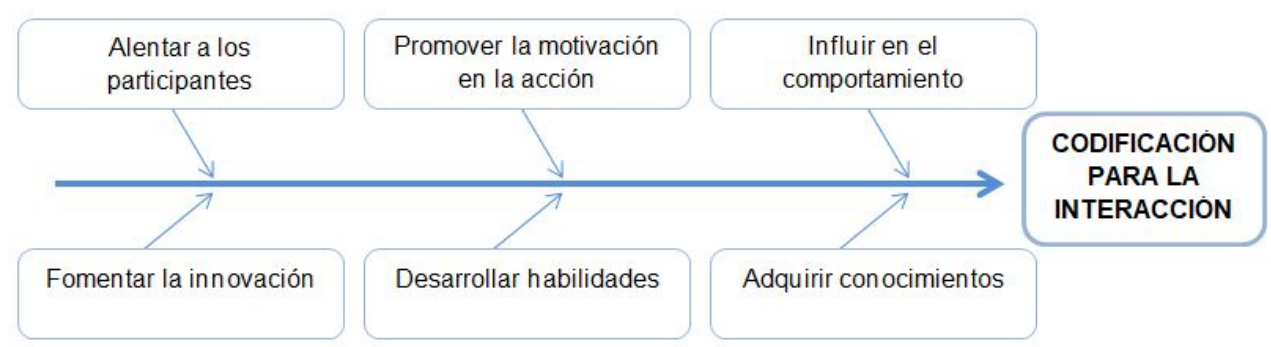

Figura 1. Categorías matrices adoptadas para el análisis de las interacciones, a partir de Kapp, Blair y Mesch (2014)

La parte final del estudio se dedica al análisis de las estrategias de juego adoptadas en las distintas propuestas $(\mathrm{N}=15)$. Este proceso nos informa de cuáles son las preferencias y afinidades de los futuros docentes y en qué dinámica del juego se sienten más seguros, predispuestos, y motivados. Para ello, se utiliza una taxonomía referida a los elementos constituyentes de la gamificación en el aprendizaje de Ortiz-Colón et al. (2018). Esta tiene como eje conceptual tres aspectos que adoptamos como categorías de análisis, a saber: la dinámica, la mecánica, y los componentes. En este caso, la información se extrae del análisis de los informes escritos elaborados por los futuros maestros, para lo cual se ha utilizado una tabla de observación y registro de frecuencias (véase Anexo 2), estableciendo una triangulación entre los datos de la observación, la puesta en práctica de la actividad en el contexto escolar y la información del informe elaborado por los grupos de trabajo de los universitarios.

\section{Exposición de resultados}

\subsection{Interacción entre los participantes}

En relación con las interacciones (inter e intra grupales: entre escolares, entre maestros en formación, entre maestros en formación y escolares) identificadas en el conjunto de actividades planeadas, y su correlación con el tipo de contenido curricular trabajado, el análisis y codificación de la acción se organiza en función de la taxonomía de seis categorías matrices anunciadas anteriormente. Sigue una descripción que argumenta y justifica los criterios adoptados para la extracción de los códigos expuestos en la Tabla 3.

a. Refuerzo del aprendizaje: Los códigos de interacción obtenidos hacen referencia especialmente a acciones comunicativas entre participantes a través de las cuales se promueve la implicación o engagement que se sostiene en el tiempo. En las acciones codificadas la interacción verbal se refuerza y los aprendizajes se exteriorizan a medida que la actividad se desarrolla.

b. Impulso de la acción: Las instrucciones sobre las pautas de la actividad y de cómo darle continuidad son en su mayoría unidireccionales (del maestro en formación a escolares). Pero se producen igualmente interacciones a remolque de las dificultades de comprensión y de las peticiones de ayuda o aclaración. Principalmente, se codifican las acciones en torno a la manipulación de los instrumentos musicales u objetos diseñados, la gestión de las 
reglas o instrucciones de uso.

c. Modificación conductual: Los códigos creados responden a interjecciones de aprobación y desaprobación, que sirven a su vez para crear o mostrar expectación, promover la competitividad y mostrar sorpresa o agrado.

d. Promoción de la innovación: Se codifican instrucciones o diálogos que impulsan la iniciativa de los escolares y se promueve de alguna forma su creatividad.

e. Desarrollo de habilidades: Se identifican situaciones donde se planifica la acción, se ejecuta una estrategia o se buscan soluciones y se coopera entre los participantes.

f. Aporte de conocimiento: Se circunscribe a la identificación de situaciones en las que aparecen los contenidos clave de la actividad, o bien conceptos derivados de su desarrollo, momentos de evaluación de los participantes, y valoración de la actividad en su conjunto.

Tabla 3. Resultado de la codificación de la interacción de los participantes, agrupadas en las seis categorías prestablecidas (Kapp, Blair y Mesch, 2014)

\begin{tabular}{|l|l|}
\hline Categorías matrices & Códigos de interacción \\
\hline $\begin{array}{l}\text { Refuerzo del aprendizaje } \\
\text { (Dinámica activa que alienta a la participación) }\end{array}$ & $\begin{array}{l}\text { Hacer preguntas } \\
\text { Dar respuesta } \\
\text { Ofrecer feedback } \\
\text { Dar la bienvenida } \\
\text { Mostrar empatía }\end{array}$ \\
\hline & $\begin{array}{l}\text { Seguir instrucciones } \\
\text { Pedir ayuda } \\
\text { Jugar con recursos musicales } \\
\text { Entregar objetos } \\
\text { (Acción motivadora) }\end{array}$ \\
$\begin{array}{l}\text { Sugerir recursos } \\
\text { Modifica la conducta } \\
\text { (Influencia de algún modo el comportamiento } \\
\text { de los participantes) }\end{array}$ & $\begin{array}{l}\text { Aprobar o rechazar la acción } \\
\text { Crear expectativa } \\
\text { Promover competitividad } \\
\text { Crear sorpresa }\end{array}$ \\
\hline $\begin{array}{l}\text { Fomento de la innovación } \\
\text { (Acciones vinculadas a la creatividad) }\end{array}$ & $\begin{array}{l}\text { Ser creativo } \\
\text { Diseñar una actividad }\end{array}$ \\
\hline $\begin{array}{l}\text { Desarrollo de habilidades } \\
\text { (Búsqueda e implementación de soluciones) }\end{array}$ & $\begin{array}{l}\text { Hacer un plan } \\
\text { Ejecutar una estrategia } \\
\text { Encontrar soluciones } \\
\text { Cooperar }\end{array}$ \\
\hline $\begin{array}{l}\text { Aporte de conocimiento } \\
\text { (Aparición de conceptos curriculares) }\end{array}$ & $\begin{array}{l}\text { Conceptualizar } \\
\text { Reflexión conjunta } \\
\text { Evaluar }\end{array}$ \\
\hline
\end{tabular}

\subsection{Aprendizajes musicales observados}

La investigación se propone determinar si la adopción de componentes lúdicos en la didáctica musical permite, a su vez, definir e identificar con claridad los componentes disciplinares propios de la formación musical propuesta. Se trata de contenidos que, en primer lugar, han sido discutidos, seleccionados, y, por tanto, asimilados por los futuros docentes, paralelamente a la reflexión y elaboración de la estrategia didáctica ludificada.

En la tabla resumen [4] se relacionan los aprendizajes musicales promovidos en las actividades, vinculados a los apartados curriculares, en correspondencia con el documento para el despliegue competencial del área (Generalitat de Catalunya, 2016). El análisis interrelaciona pues las acciones promovidas en el alumnado con elementos disciplinares y los conceptos musicales que sustentan el aprendizaje durante la práctica. El resultado es el de una diversidad de aprendizajes, en la que cada categoría codificada queda vinculada a una variedad notable de elementos que articulan las actividades. 
Tabla 4. Resultado del análisis de las actividades musicales diseñadas y los objetivos de aprendizaje propuestos

\begin{tabular}{|l|l|}
\hline Ítems curriculares & Objetivos de aprendizaje vinculados a las actividades \\
\hline $\begin{array}{l}\text { Vocabulario musical: instrumentos musi- } \\
\text { cales, notación, dinámica, tempo. }\end{array}$ & $\begin{array}{l}\text { Identificar timbres } \\
\text { Reconocer imágenes de instrumentos musicales } \\
\text { Leer las notas en el pentagrama } \\
\text { Interpretar unas dinámicas con la voz } \\
\text { Calificar el tempo de una música }\end{array}$ \\
\hline $\begin{array}{l}\text { Cantar y tocar: repertorio musical, técnica } \\
\text { instrumental, educación vocal y auditiva. }\end{array}$ & $\begin{array}{l}\text { Cantar una canción } \\
\text { Interpretar un ritmo } \\
\text { Reconocer una melodía }\end{array}$ \\
\hline $\begin{array}{l}\text { Música y movimiento: expresión musical, } \\
\text { coreografía creativa, coordinación. }\end{array}$ & $\begin{array}{l}\text { Aprender un baile } \\
\text { Expresar una música con el cuerpo } \\
\text { Interpretar un ritmo con percusión corporal } \\
\text { Crear un movimiento a partir del sonido }\end{array}$ \\
\hline $\begin{array}{l}\text { Crear un ritmo o melodía: improvisación } \\
\text { y escritura musicales. }\end{array}$ & $\begin{array}{l}\text { Inventar un ritmo } \\
\text { Completar una secuencia rítmico-melódica } \\
\text { Escribir ritmos dictados } \\
\text { Colocar o reordenar las notas en el pentagrama }\end{array}$ \\
\hline $\begin{array}{l}\text { Identificar patrones musicales: lectura e e } \\
\text { interpretación de elementos del lenguaje } \\
\text { musical. }\end{array}$ & $\begin{array}{l}\text { Ordenar una secuencia rítmica } \\
\text { Reproducir un ritmo } \\
\text { Asociar la partitura a la audición }\end{array}$ \\
\hline
\end{tabular}

\subsection{Estrategias de la gamificación}

En primer lugar, analizamos la dinámica como eje singular que permite clasificar la tipología de juego que se adopta. En este apartado se observan un conjunto de acciones que se encadenan de forma programada y con una finalidad preestablecida. La Figura 2 expresa los resultados del análisis de las quince actividades. Entre los diez elementos observados, obtienen los registros más altos el feedback o retroalimentación, la interacción social, y la cooperación. Este resultado respondería a la lógica de que, ante el abordaje de un reto o problema, es indispensable que los participantes formen y funcionen como equipo, que establezcan relaciones y vínculos entre ellos, al tiempo que se va creando conocimiento compartido.

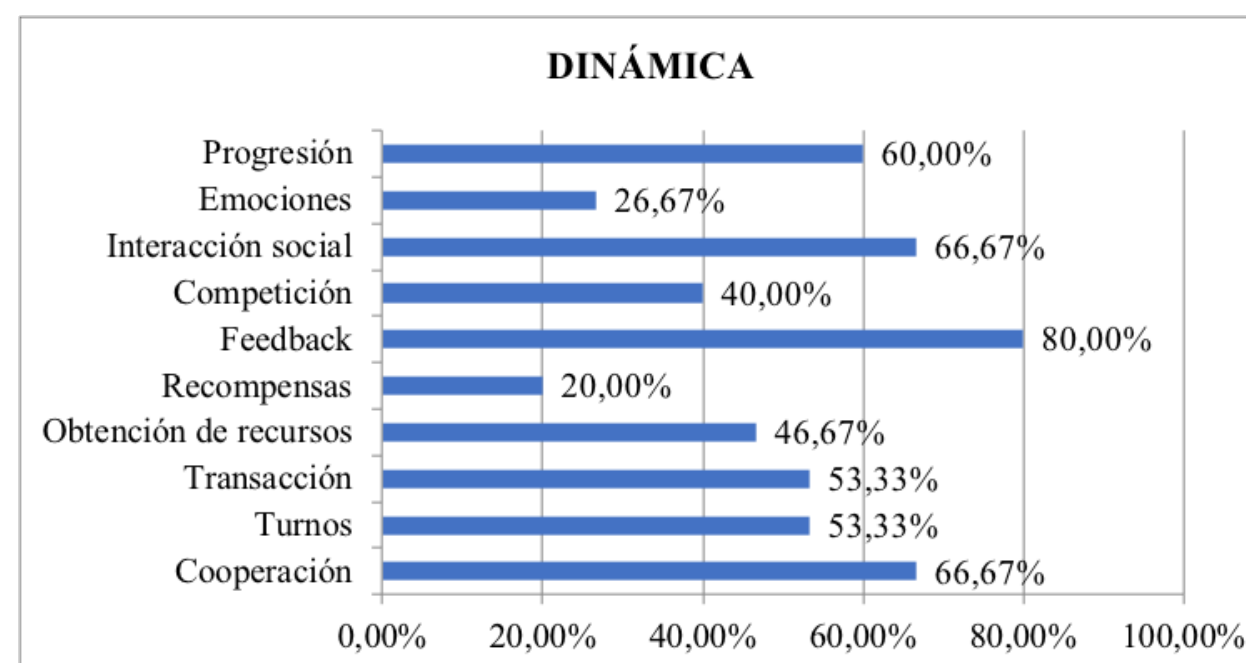

Figura 2. Frecuencia de uso de los elementos asociados a la dinámica en las actividades gamificadas $(\mathrm{N}=15)$

En segundo lugar, analizamos el ámbito del componente, aspecto que permite determinar de qué tipo son los retos que guían la acción y son compartidos por los jugadores, que deben respetar a la vez que resuelven el desafío planteado. En concreto, del conjunto de actividades sobresale, primeramente, el de las clasificaciones: a lo largo del juego se desarrollan distintas habilidades y dimensiones de la educación musical consistentes en clasificar elementos concretos (instrumentos, figuras musicales, etc.). Seguidamente, los equipos: hay un objetivo compartido por todos los jugadores y deben resolver el reto en equipo. Y, también, las búsquedas: las estrategias en este apartado se centran en encontrar o descubrir un concepto tras una pregunta o enunciado, reconocer una melodía, encontrar el orden correcto, etc. 


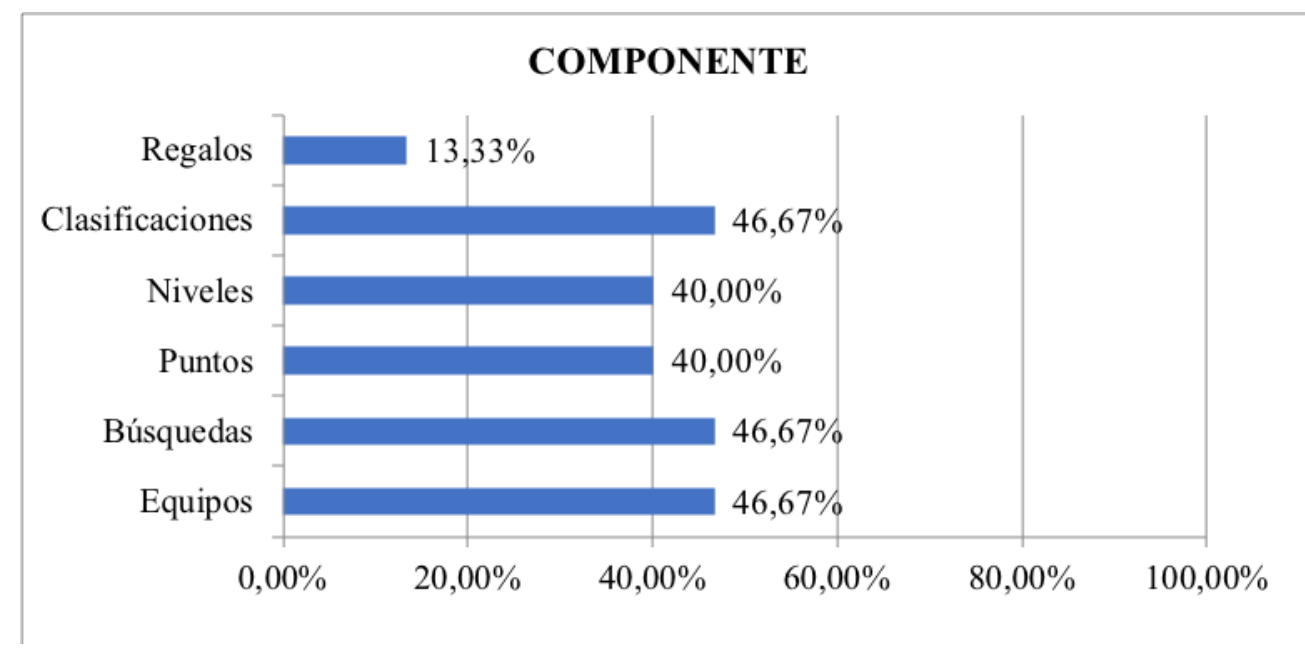

Figura 3. Frecuencia de uso de los elementos asociados al componente en las actividades gamificadas $(\mathrm{N}=15)$

Por último, el tercer ámbito de observación es el referido a la mecánica del juego. En este caso se identifican tres aspectos, que son los desafios, las insignias y los logros. Entre ellos, claramente predomina el primer elemento, aspecto directamente relacionado con el objetivo de activar la motivación de los participantes. Un desafío o reto busca de entrada un resultado inmediato, consistente en la implicación instantánea de todos los participantes. Por ello, los elementos de la mecánica, en su conjunto, deben ser comprendidos por todos los implicados. Por lo tanto, se puede inferir que elaborar y comunicar bien los elementos de la mecánica incide directamente en las competencias comunicativas del futuro docente.

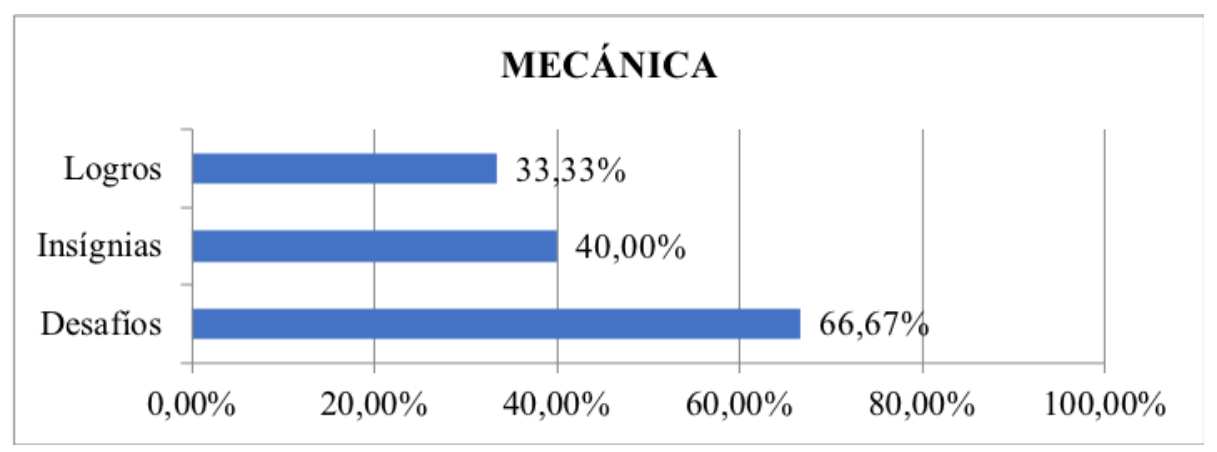

Figura 4. Frecuencia de uso de los elementos asociados a la mecánica en las actividades gamificadas $(\mathrm{N}=15)$

\section{Discusión y conclusiones}

En la revisión de fondo firmada por De Sousa, Durelli, Macedo y Isotani (2014), se subrayan tres factores principales en la conjugación de elementos del juego y objetivos de aprendizaje, a saber: a) el incremento de la motivación, b) la mejora competencial en habilidades concretas, y c) el aumento y mejora de resultados del aprendizaje. Resultados coincidentes, parcialmente, con el metaanálisis de Hamari et al., (2014), que ofrece como resultado tres categorías principales en los estudios de gamificación educativa: a) las aportaciones al estudio de la motivación, b) los efectos psicológicos, y c) los resultados conductuales. En nuestro estudio, el objetivo principal y preguntas de investigación formuladas se enmarcan en las líneas mayoritarias o mainstream de buena parte de la literatura existente. También, sin duda, la dimensión disciplinar (la música y su didáctica) tiene un peso característico y distintivo en el estudio. Por ello vamos a referirnos, a continuación, a algunos antecedentes en el campo con los que contrastar nuestros hallazgos.

Observamos, con Koops y Taggart (2010), cómo el recurso del juego mantiene una alta valoración y desarrollo didáctico en la escuela, por todos los beneficios reseñados hasta el momento. Después de nuestro estudio, en concordancia con estos autores, parecen adecuadas y relevantes las ganancias de plantear, en el contexto de la formación inicial del maestro, el trabajo del lenguaje musical bajo estrategias lúdicas que permiten abordar la relación con los conceptos disciplinares clave de una forma socializada, práctica y creativa. En la misma línea, parece lógico observar que aligerar el nivel curricular de la materia, por un lado, permite, por el otro, reforzar la confianza e interés del futuro docente por su aplicación final en el aula.

Este tipo de práctica, en colaboración con la escuela y de aplicación final de propuestas con los alumnos destinatarios, se presenta como una excelente ocasión para desarrollar competencias profesionales específicas. El futuro maestro se prepara y se sumerge en un contexto profesional donde la ludificación es cotidiana y bienvenida, también 
desde la práctica diaria en el aula de música. Morante y Castellano (2019), por ejemplo, han reportado cómo, además del propio aprendizaje musical, con la experiencia lúdico-musical los escolares de Primaria desarrollan capacidades de "alta significancia", flexibilizándose a su vez, las fronteras y divisiones entre las diferentes áreas del currículo. Podemos inferir que los futuros maestros experimentan beneficios equivalentes en el abordaje lúdico de la didáctica musical y la elaboración de actividades como las que se han evaluado en este artículo, pues se promueven principalmente habilidades prácticas consistentes en resolver preguntas, retos y dificultades de diversa naturaleza. Es también un contexto adecuado y el marco de actuación propio de una instrucción universitaria competencial, es decir, de una formación aplicada donde se combinan de forma paritaria un conjunto de saberes (Zabala \& Arnau, 2014).

En respuesta a las tres preguntas formuladas, diremos sobre la interacción entre los participantes (en los roles de alumno de grado y escolar de Primaria), que los resultados refuerzan la idea sobre una relación bidireccional, en la cual predomina el registro interrogativo y un diálogo breve y paritario. En la línea de la visión creativo-musical dialógica entre docente y alumno de nuestro añorado Marcelo Giglio, se pone de manifiesto cómo estrategias y dinámicas próximas al juego incrementan la perspectiva colaborativa del proceso formativo (Giglio, 2013).

Por otro lado, la investigación demuestra que la gamificación en los grados de Magisterio es una opción apta y efectiva para abordar una aplicación práctica de los contenidos musicales básicos que debe conocer el futuro maestro generalista, cuya relación con la disciplina está marcada con frecuencia por una baja autopercepción de dominio y eficacia (Holden \& Button, 2006). A la vez, se fomenta la iniciativa y creatividad de los estudiantes, promoviendo la conexión con la realidad social, su pensamiento crítico y su vinculación con su futuro contexto profesional. Resultados paralelos a los expuestos por Van As y Excell (2018), en cuyo caso, a partir de una muestra de futuros docentes de educación infantil, la didáctica musical resultaba mediadora para promover una mejor predisposición de estos hacia la dinámica del juego en el aula.

Por último, a partir de los resultados obtenidos, se aprecia que el aprendizaje musical basado en el juego, según las estrategias desarrolladas en la práctica analizada, promueve en los escolares receptores de las actividades la autorregulación del aprendizaje. Y lo hace, según lo visto, de forma mediatizada por la potenciación de dinámicas de feedback y el estímulo de habilidades sociales colaborativas.

\section{Aplicación y limitaciones del estudio}

En el artículo se abordan las relaciones entre educación musical y juego a partir de un caso aplicado a la formación musical del maestro de Primaria en formación, cuyos resultados y conclusiones resultan pertinentes como referentes en otros contextos docentes. El trabajo de fundamentación y análisis conceptual realizado desvelan, de entrada, una diversidad terminológica en torno al constructo denominado "aprendizaje lúdico". Consideramos clarificador y útil, tanto en la investigación reportada como en un futuro desarrollo, reflexionar y ejemplarizar, en el ámbito de la educación musical, conceptos clave tales como la gamificación, la ludificación, el juego, la simulación, etc. Estrategias y recursos que, en su conjunto, se valoran adecuados en procesos cuyos objetivos transformacionales pasen por incidir en la motivación y el empoderamiento de los participantes, el establecimiento de dinámicas colaborativas, y la activación de procesos de feedback entre alumno y docente.

Las limitaciones de los resultados expuestos son los propios del estudio de caso y del paradigma cualitativo, es decir, su transferibilidad y generalización. Se requerirían otros informes sobre prácticas paralelas a la analizada aquí, ampliando a su vez la muestra y abordando el tema, por ejemplo, desde una perspectiva transversal, o comparada, entre diferentes programas y distintas etapas educativas.

\section{Referencias bibliográficas}

Ardila-Muñoz, J. Y. (2019). Supuestos teóricos para la gamificación de la educación superior. Magis, Revista Internacional de Investigación en Educación, 12(24), 71-84. http://doi.org/10.11144/javeriana.m12-24.stge

Bautista, J., \& Guzmán, D. (2003). Jugar, aprender y sus contradicciones en la sociedad del conocimiento. Comunicación y Pedagogía. Nuevas Tecnologías y Recursos Didácticos, 186, 75-81. Disponible en: https://bit.ly/3hqcJvd

Borrás, O. (2015). Fundamentos de la gamificación. Madrid: GATE-Universidad Politécnica de Madrid. Disponible en: https:// bit.ly/3fchuWx

Burnard, P. (2012). Musical creativities in practice. Oxford: Oxford University Press.

Cain, T. (2013). Investigación-acción en educación musical. En M. Díaz \& A. Giráldez (Coords.), Investigación cualitativa en educación musical (pp. 57-75). Barcelona: Graó.

Carrión, E. (2019). El uso del juego y la metodología cooperativa en la Educación Superior: una alternativa para la enseñanza creativa. Artseduca, 23, 70-97. http://dx.doi.org/10.6035/Artseduca.2019.23.4

Catalunya (2015). "Decret 119/2015, de 23 de juny, d'ordenació dels ensenyaments de l'educació primària". Diari Oficial de la Generalitat de Catalunya (26 juny 2015), núm. 6900, p. 1-136. Disponible en: https://bit.ly/3tN2hAI

Clapper, T. (2014). Situational interest and instructional design: a guide for simulation facilitators. Simulation and gaming 45(2), 167-182. http://doi.org/10.1177/1046878113518482 
Cleaver, D., \& Ballantyne, J. (2013). Teachers' views of constructivist theory: A qualitative study illuminating relationships between epistemological understanding and music teaching practice. International Journal of Music Education, 32(2), $228-241$. http://doi.org/10.1177/0255761413508066

Contreras, R.S. (2017). Gamificando en contextos educativos. Revisando literatura para aclarar conceptos. En R.S. Contreras \& J.L. Eguia (Eds.), Experiencias de gamificación en las aulas (pp. 11-17). Bellaterra, España: Institut de la Comunicació, Universitat Autònoma de Barcelona. Disponible en: https://bit.ly/3y2JtR8

Creswell, J.W., \& Creswell, J. D. (2018). Research Design: Qualitative, Quantitative, and Mixed Methods Approaches. London: SAGE.

Criss, E. (2010). Teamwork in the Music Room. Music Educators Journal, 97, 30-36. http://doi.org/10.1177/0027432110380550

Csikszentmihalyi, M. (1990). Flow, the psychology of optimal experience. New York: Harper \& Row.

Csikszentmihalyi, M. (2000). Beyond boredom and anxiety: Experiencing flow in work and play (25 th anniversary ed.). San Francisco, CA: Jossey-Bass.

De Sousa, S., Durelli, V., Macedo H., \& Isotani, S. (2014). A systematic mapping on gamification applied to education. En Proceedings of the $29^{\text {th }}$ Annual ACM Symposium on Applied Computing (SAC '14) (pp. 216-222). https://doi.org/10.1145/2554850. 2554956Deterding, S. (2013). Gameful Design for Learning. $T+D, 67(7), 60-63$. Disponible en: https://bit.ly/33CHWTP

Deterding, S. (2014). The Ambiguity of Games: Histories and Discourses of a Gameful World. En Steffen P. Walz \& Sebastian Deterding (eds.), The Gameful World. Approaches, Issues, Applications (pp. 23-64). Cambridge, MA: MIT Press.

Deterding, S., Dixon, D., Khaled, R., \& Nacke, L. (2011). From game design elements to gamefulness: defining "gamification." En Proceedings of the $15^{\text {th }}$ International Academic MindTrek Conference: Envisioning Future Media Environments (MindTrek '11). Association for Computing Machinery, New York, NY, USA, 9-15. http://doi.org/10.1145/2181037.2181040

Elkind D. (2008). Can we play? Greater Good Magazine, 4(2), 14-17. Disponible en: https://bit.ly/3w37zJU

García-Hernández, R.J., Barbancho, I., Tardón, L.J., Arrambarri, J., Magdics, M., \& Sbert, M. (2014). E-cecilia: implementation of a music game. Proceedings of CoSECivi 2014 Computer Science. Disponible en: https://bit.ly/3fbPXV5

García-Lázaro, I. (2019). Escape Room como propuesta de gamificación en educación. Revista Educativa Hekademos, 27, 71-79. Disponible en: https://dialnet.unirioja.es/servlet/articulo?codigo $=7197820$

Garris, R., Ahlers, R., \& Driskell, J. E. (2002). Games, motivation, and learning: A research and practice model. Simulation \& Gaming, 33, 441-467. http://doi.org/10.1177/1046878102238607

Gault, B.M. (2016). Kodály-inspired teaching: a bridge to musical fluency. En G.M. Abril \& B.M. Gault (Eds.), Teaching General Music: Approaches, Issues, and Viewpoints (pp. 73-88). New York: Oxford University Press. http://doi.org/10.1093/acprof:oso/9780199328093.001.0001

Generalitat de Catalunya (2016). Competències bàsiques de l'Educació Artística. Educació Artística: visual i oord.a, música i dansa. Identificació i desplegament a l'Educació Primària. Barcelona: Departament d'Ensenyament. Disponible en: https:// bit.ly/3hmEAMH

Giglio, M. (2013). Cuando la colaboración creativa cambia la forma de enseñar. Santander: Ediciones Universidad Cantabria.

Gomes, C., Figueiredo, M., \& Bidarra, J. (2014). Gamification in Teaching Music: Case Study. En EduRe'14 Conference Proceedings. Universidad Politécnica de Valencia. Disponible en: https://bit.ly/3tHoVtZ

Gomes, J., Figueiredo, M., \& Amante, L. (2014). Musical Journey: A virtual world gamification experience for music learning. International Journal on Advances in Education Research, 1(1), 1-21. Disponible en: http://hdl.handle.net/10400.1/9139

Hamari, J., Koivisto, J., \& Sarsa, H. (2014). Does Gamification Work? - A Literature Review of Empirical Studies on Gamification. Proceedings of the 47 th Hawaii International Conference on System Sciences, Hawaii, USA, January 6-9: 3025-3034. Disponible en: https://url2.cl/cAb3n

Hamari, J., \& Koivisto, J. (2014). Measuring Flow in Gamification: Dispositional Flow Scale-2. Computers in Human Behavior, 40, 133-134. http://doi.org/10.1109/HICSS.2014.377

Hassinger-Das, B., Toub, T. S., Zosh, J. M., Michnick, J., Golinkoff, R., \& Hirsh-Pasek, K. (2017). More than just fun: a place for games in playful learning. Infancia y Aprendizaje, 40(2), 191-218. http://doi.org/10.1080/02103702.2017.1292684

Hein, E. (2014). Music games in education. En K. Schrier (Ed.), Learning, Education and Games (pp. 93-108). Pittsburgh: ETC Press. https://bit.ly/3tGAx0r

Hemsy de Gainza, V. (2004). La educación musical en el siglo XX. Revista Musical Chilena, 201, 74-81. http://doi.org/10.4067/ S0716-27902004020100004

Hirsh-Pasek, K., \& Golinkoff R.M. (2008). Why Play = Learning. En Tremblay RE, Boivin M, \& Peters RdeV, (eds). Smith PK, (topic ed). Encyclopedia on Early Childhood Development. Disponible en: https://bit.ly/3y3Sb1r

Hirsh-Pasek, K., Zosh, J. M., Golinkoff, R. M., Gray, J. H., Robb, M. B., \& Kaufman, J. (2015). Putting education in "educational" apps: Lessons from the science of learning. Psychological Science in the Public Interest, 16, 3-34. http://doi. org/10.1177/1529100615569721

Holden, H., \& Button, S.W. (2006). The teaching of music in the primary school by the non-music specialist. British Journal of Music Education, 23(1), 23-38. http://doi.org/10.1017/S0265051705006728

Johnson, L., Adams Becker, S., Estrada, V., \& Freeman, A. (2014). NMC Horizon Report: 2014 K-12 Edition. Austin: The New Media Consortium. Disponible en: https://bit.ly/2RNnIEe

Kapp, K. M. (2012). The Gamification of learning and instruction: game-based methods and strategies for training and education. San Francisco: Pheiffer. 
Kapp, K. M., Blair, L., \& Mesch, R. (2014). The Gamification of learning and instruction. Ideas into practice. San Francisco: Wiley.

Koops, L.H., \& Taggart, C.C. (2010). Learning through play: Extending an early childhood music education approach to undergraduate and graduate music education. Journal of Music Teacher Education, 20(2), 55-66. http://doi.org/10.1177/1057083710373578

Lieberoth, A. (2015). Shallow Gamification, Testing Psychological Effects of Framing an Activity as a Game. Games and Culture, 10(3), 229-248. http://doi.org/10.1177/1555412014559978

Lozada, C., \& Betancur S. (2017). La gamificación en la educación superior: una revisión sistemática. Revista Ingenierías Universidad de Medellín, 16(31), 97-124. https://doi.org/10.22395/rium.v16n31a5

Lucato, M. (2001). El método Kodály y la formación del profesorado de música. Revista electrónica LEEME, 7, 1-7. Disponible en: https://ojs.uv.es/index.php/LEEME/article/view/9725

Margoudi, M., Oliveira, M., \& Waddell, G. (2016). Game-Based Learning of Musical Instruments: A Review and Recommendations. Proceedings of the $10^{\text {th }}$ European Conference on Games Based Learning (pp. 426-433), Paisley, Scotland. Disponible en: https://bit.ly/3oamX4c

Margoudi, M., Waddell, G., \& Oliveira, M. (2017). Co-creating a gamified solution for music learning. Proceedings of the 11 ${ }^{\text {th }}$ European Conference on Game-Based Learning (pp. 420-427), Graz, Austria. Disponible en: https://bit.ly/33F7Sy5

Morante, P.C., \& Castellano, I. (2019). Re-creando: propuesta de estrategias lúdico musicales para promover aprendizajes creativos y significativos en la escuela. En Manuel Roberto y Enrique Soria (coords.), Memorias del quinto Congreso Internacional de Ciencias Pedagógicas de Ecuador: Aprendizaje en la sociedad del conocimiento: modelos, experiencias y propuestas, (pp.1005-1014). Disponible en: https://bit.ly/3y6j59a

Navarro, J. L. (2017). Pautas para la aplicación de métodos de enseñanza musical desde un enfoque constructivista. Revista Electrónica de Investigación Educativa, 19(3), 143-160. http://doi.org/10.24320/redie.2017.19.3.675

Ortiz-Colón, A.M., Jordán, J., \& Agredal, M. (2018). Gamificación en educación: una panorámica sobre el estado de la cuestión. Educação e Pesquisa, 44, 1-17. http://doi.org/10.1590/s1678-4634201844173773

Perdomo, I.R., \& Rojas, J.A. (2019). La ludificación como herramienta pedagógica: algunas reflexiones desde la psicología. REXE. Revista de Estudios y Experiencias en Educación, 18(36), 161-175. http://doi.org/10.21703/rexe.20191836perdomo9

Pesek, M., Vucko, Z., Savli, P., Kavcic, A., \& Marolt, M. (2020). Troubadour: A gamified e-learning platform for ear training. IEEE Access, 8, 97090-97102. http://doi.org/10.1109/ACCESS.2020.2994389

Politis, D., Margounakis, D., Aleksii, V., \& Karanikas, N. (2017). From Music Gamification to the Musification of Games: A Synaesthetic Learning Pathway. International Journal of New Technologies in Science and Engineering, 4(1), 14-27. Disponible en: https://bit.ly/3tHtDb9

Prieto, J.M. (2020). Una revisión sistemática sobre gamificación, motivación y aprendizaje en universitarios. Teoría de la Educación. Revista Interuniversitaria, 32(1), 73-99. http://doi.org/10.14201/teri.20625

Ray, J.A. (2008). Constructivism and classroom teachers: What can early childhood teacher educators do to support the constructivist journey? Journal of Early Childhood Teacher Education, 23(4), 319-325. http://doi.org/10.1080/1090102020230404

Restrepo, B. (2006). Tendencias actuals en la educación superior: rumbos del mundo y rumbos del país. Revista Educación y Pedagogía, 18(46), 79-90.

Río Sadornil, D. (2003). Métodos de investigación en educación. Vol. 1: Proceso y diseños no complejos. Madrid: UNED.

Robson, K., Plangger, K., Kietzmann, J., McCarthy, I., \& Pitt, L. (2015). Is it all a game? Understanding the principles of gamification. Business Horizons, 58(4), 411-420. http://doi.org/10.1016/j.bushor.2015.03.006

Saldana, J. (2016). The coding manual for qualitative researchers $\left(3^{\text {rd }}\right.$ ed.). London: SAGE.

Scott, Sh. (2009). A minds-on approach to active learning in general music. General music today 24(1), 19-26. http://doi.or$\mathrm{g} / 10.1177 \% 2 \mathrm{~F} 1048371309354432$

Scott, Sh. (2011). Contemplating a constructivist stance for active learning within music education. Arts education policy review, 112(4), 191-198. http://doi.org/10.1080/10632913.2011.592469

Shively, J. (2015). Constructivism in Music Education. Arts Education Policy Review, 116(3), 128-136. http://doi.org/10.1080/1 0632913.2015 .1011815

Singer, D., Golinkoff, R. M., \& Hirsh-Pasek, K. (Eds.). (2006). Play = learning: How play motivates and enhances children's cognitive and social-emotional growth. New York: Oxford University Press. http://doi.org/10.1093/acprof:oso/9780195304381.001.0001

Teixes, F. (2014). Gamificación: Fundamentos y Aplicaciones. Barcelona: UOC, Business School.

Tulloch, R., \& Randell-Moon, H.E.K. (2018). The politics of gamification: Education, neoliberalism and the knowledge economy. Review of Education, Pedagogy, and Cultural Studies, 40(3), 204-226. https://doi.org/10.1080/10714413.2018.1472484

Van As, A.J., \& Excell, L. (2018). Strengthening early childhood teacher education towards a play-based pedagogical approach through a music intervention programme, South African Journal of Childhood Education 8(1), a525. http://doi.org/10.4102/ sajce.v8i1.525

Wagner, C. (2017). Digital gamification in private music education. Antistasis, 7(1), 115-122. Disponible en: https://journals.lib. unb.ca/index.php/antistasis/article/view/24904

Weisberg, D.S., Hirsh-Pasek, K., Golinkoff, R.M., Kittredge, A.K., \& Klahr, D. (2016). Guided play: Principles and practices. Current Directions in Psychological Science, 25, 177-182. http://doi.org/10.1177/0963721416645512

Werbach, K., \& Hunter, D. (2014). Gamificación: revoluciona tu negocio con las técnicas de los juegos. Madrid: Pearson Educación. 
Zabala, A., \& Arnau, L. (2014). Métodos para la enseñanza de las competencias. Barcelona: Graó

Zich, I., Ortega-Ruiz, R., \& Sibaja, S. (2016). Children's play and affective development: affect, school adjustment and learning in preschoolers. Infancia y Aprendizaje, 39, 380-400. http://doi.org/10.1080/02103702.2016.1138718

\section{ANEXO 1}

\begin{tabular}{|c|c|c|c|}
\hline \multicolumn{4}{|c|}{ Evaluación de la práctica: "Actividades ludificadas de aprendizaje musical" } \\
\hline Trabajo desarrollado & Ítems evaluados & Descriptor & Valor de 1 a 10 \\
\hline \multirow{8}{*}{ Puesta en práctica } & \multirow{3}{*}{ Aplicación de la actividad } & La puesta en práctica es original y creativa & \\
\hline & & $\begin{array}{l}\text { El grupo de trabajo mantiene una buena } \\
\text { coordinación interna }\end{array}$ & \\
\hline & & $\begin{array}{l}\text { Las herramientas, técnicas y recursos utili- } \\
\text { zados son variados }\end{array}$ & \\
\hline & \multirow{3}{*}{ Dominio disciplinar } & $\begin{array}{l}\text { Se prioriza el trabajo con elementos de } \\
\text { lenguaje musical }\end{array}$ & \\
\hline & & $\begin{array}{l}\text { Se adecua la actividad a los contenidos } \\
\text { relacionados con la etapa educativa del } \\
\text { alumnado }\end{array}$ & \\
\hline & & $\begin{array}{l}\text { Se aborda una visión inclusiva de la edu- } \\
\text { cación artística }\end{array}$ & \\
\hline & \multirow{2}{*}{$\begin{array}{l}\text { Interacción con los alum- } \\
\text { nos de Primaria }\end{array}$} & $\begin{array}{l}\text { Todos los miembros del grupo interactúan } \\
\text { con los alumnos }\end{array}$ & \\
\hline & & $\begin{array}{l}\text { Se refuerza el protagonismo de los alum- } \\
\text { nos con el desarrollo de la actividad }\end{array}$ & \\
\hline \multirow{8}{*}{ Documento escrito } & Objetivos & $\begin{array}{l}\text { Los objetivos están bien redactados y son } \\
\text { específicos de la actividad }\end{array}$ & \\
\hline & Contenidos & $\begin{array}{l}\text { Los contenidos están bien escogidos, son } \\
\text { coherentes con la actividad y el nivel de } \\
\text { los alumnos }\end{array}$ & \\
\hline & Competencias básicas & $\begin{array}{l}\text { Se trabaja alguna de las ocho competen- } \\
\text { cias básicas de la etapa educativa (Prima- } \\
\text { ria) }\end{array}$ & \\
\hline & $\begin{array}{l}\text { Ámbitos y dimensiones } \\
\text { curriculares }\end{array}$ & $\begin{array}{l}\text { Se concretan los ámbitos y dimensiones } \\
\text { específicas de educación artística de forma } \\
\text { coherente con la actividad }\end{array}$ & \\
\hline & Metodología & $\begin{array}{l}\text { Se definen los principales elementos de } \\
\text { gamificación de la actividad }\end{array}$ & \\
\hline & Criterios de evaluación & $\begin{array}{l}\text { Se han elaborado criterios de evaluación y } \\
\text { son coherentes con la actividad propuesta }\end{array}$ & \\
\hline & \multirow{2}{*}{ Descripción de la actividad } & $\begin{array}{l}\text { Se describe de forma comprensible y es- } \\
\text { tructurada }\end{array}$ & \\
\hline & & $\begin{array}{l}\text { Detallan los materiales necesarios y su } \\
\text { sentido didáctico }\end{array}$ & \\
\hline \multicolumn{4}{|l|}{ Suma total } \\
\hline Puntuación media & & & \\
\hline
\end{tabular}




\section{ANEXO 2}

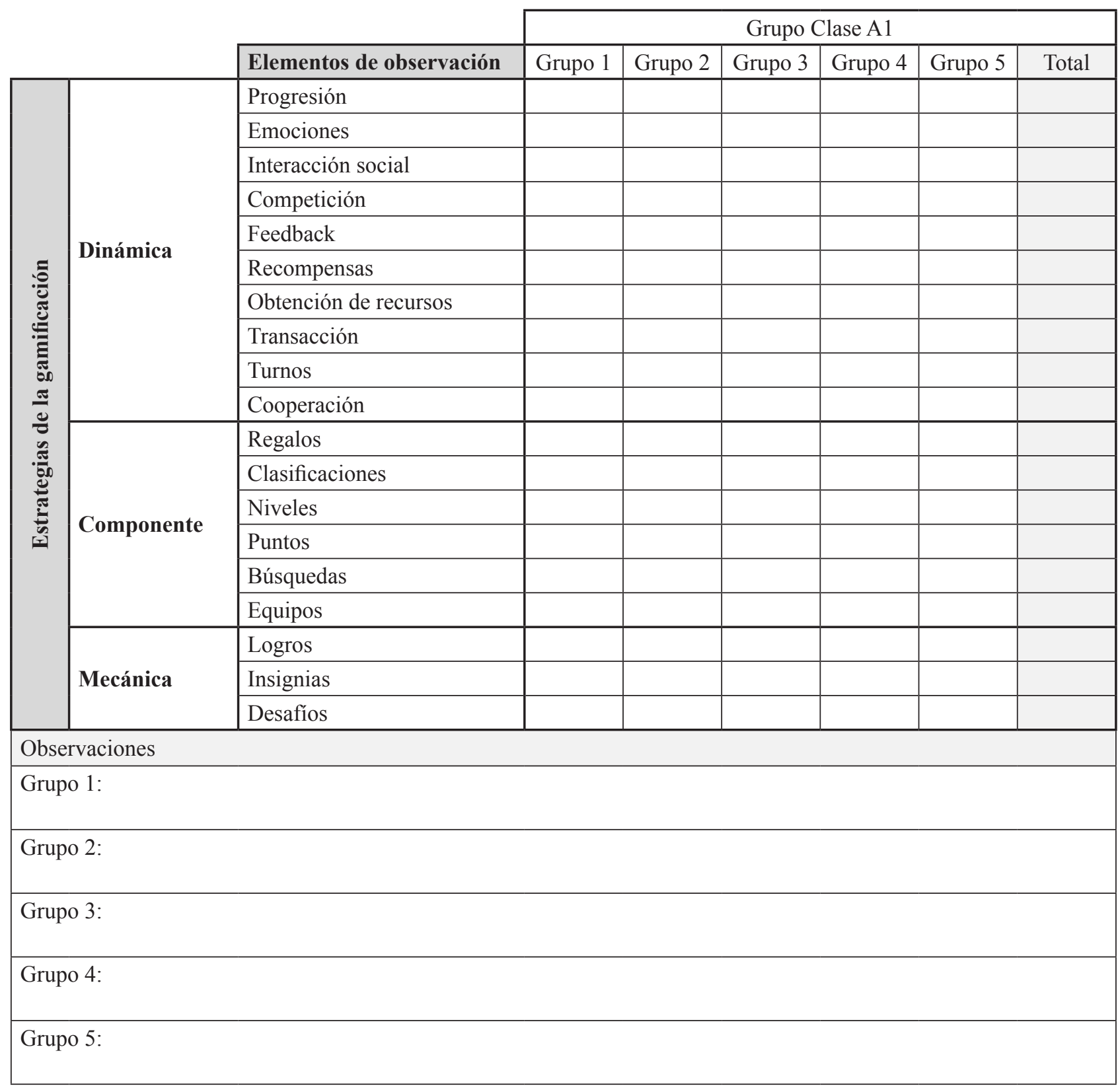

